\title{
Tracking Control for Snake Robot Joints
}

\author{
Aksel A. Transeth, Nathan van de Wouw, Alexey Pavlov, João P. Hespanha, and Kristin Y. Pettersen
}

\begin{abstract}
This paper considers the problem of model based control of the joints of a snake robot without wheels. The potential range of applications for snake robots are numerous, and delicate operations such as inspection and maintenance in industrial environments or performing search and rescue operations require precise control of a snake robot joints. To this end we present a controller that asymptotically stabilizes the joints of the snake robot to a desired reference trajectory. The controller is based on input-output linearization of a control plant model of the snake robot dynamics also developed in this paper. In addition, we provide a formal Lyapunov-based proof of the closed-loop stability, together with simulation results for a smooth dynamical model. Finally, the performance of the controller is tested on a non-smooth snake robot model with set-valued Coulomb friction that offers an accurate description of the stick-slip transitions during locomotion.
\end{abstract}

\section{INTRODUCTION}

Snake robots have the potential of contributing vastly in areas such as rescue missions, surgery, fire-fighting, and maintenance. The highly articulated body allows the snake robot to traverse difficult terrains such as collapsed buildings or the chaotic environment caused by a car collision in a tunnel. The snake robot can also inspect narrow passages or complex structures in possibly hazardous areas of industrial plants. All these tasks demand accurate control of the snake robot joints. This control problem is the subject of this paper.

Research on snake robots has increased the past ten to fifteen years and many new results on motion patterns, snake robot design, and control have been presented [1]. Many authors base the choice and implementation of the most common serpentine motion pattern 'lateral undulation' on the serpenoid curve found in [2]. This is a curve that describes the motion of a biological snake while moving by lateral undulation, and a snake robot can follow an approximation to this curve by setting its joint angles according to a sine-curve that is phase-shifted between adjacent joints. This approach to snake robot locomotion has been widely implemented for snake robots that have either wheels [3], [4] or a friction property such that each link of the snake robot glides easier forward and backward compared to transversal motion [5], [6]. A no-slip constraint (i.e. a non-holonomic velocity constraint) on each wheel is sometimes introduced in the mathematical model, thus avoiding that a link slips sideways. Such an approach is presented in [3] where the no-slip constraint allows one to significantly reduce the model. A Lyapunovbased proof for this reduced model together with a proposed controller shows that the snake robot is able to move to a position reference. A velocity controller for a wheel-less snake robot with the friction property described above, is

A. A. Transeth, A. Pavlov, and K. Y. Pettersen are with the Department of Engineering Cybernetics at the Norwegian University of Science and Technology, NO-7491 Trondheim, Norway.

(e-mail: Aksel.Andreas. Transeth@itk.ntnu.no)

A. A. Transeth is also with the Department of Applied Cybernetics at SINTEF IKT, NO-7465 Trondheim, Norway.

N. v. d. Wouw is with the Department of Mechanical Engineering, Eindhoven University of Technology, POBox 513, 5600 MB Eindhoven, The Netherlands.

J. P. Hespanha is with the Center for Control Engineering and Computation,University of California, Santa Barbara, CA 93106-9560 USA presented in [5]. The simulation and experimental results presented in that paper indicate that the snake robot is able to stay within a reasonable offset of the desired speed. However, no formal stability analysis of the closed-loop system is given. Significant results on controllability and observability of the snake robot are presented in [7]-[9]. However, these results are only based on a kinematic description of snake robot locomotion and thus do not include the dynamics of the motion. For wheel-less snake robots it is not sufficient to consider a purely kinematic model for the motion pattern lateral undulation, as the friction between the snake robot and the ground surface is essential for locomotion. Therefore, the friction needs to be considered for wheel-less snake robots, and this motivates including the dynamics into the stability analysis of the snake robot.

In this paper we consider a snake robot without wheels and propose a controller for its joints that asymptotically stabilizes a desired motion pattern for the snake robot. Notice that an accurate model of the friction between the snake robot and the ground surface typically is, together with the remaining dynamics of the snake robot, nonlinear and the control of such a model requires nonlinear control techniques. More specifically, a set-valued force law is needed to accurately describe directional Coulomb friction with stickslip transitions during motion. See [10]-[13] for more details on the advantages of modeling with set-valued force laws. The nonlinear control techniques (input-output linearization techniques [14], [15]) employed in this paper, however, are generally only fit to cope with smooth nonlinear systems. In order to make the control problem tractable, we approximate the Coulomb friction with a smooth friction model and design a nonlinear control law for the resulting smooth system. The framework for modeling robot manipulators can be used to model a snake robot. We reformulate the standard dynamics of a robot manipulator and develop a control plant model to aid the control design with a formal Lyapunovbased stability proof which we present. This extends the results from [5] where the control design is based on only a (linear) viscous friction model without a formal stability proof. In addition, we show by simulations that our proposed controller is also effective for a snake robot model with setvalued Coulomb friction. Please note that we do not give a reference for the global position and orientation of the snake robot which is beyond the scope of this paper, instead we focus on proving that each joint is able to track a desired reference angle.

This paper is organized as follows. Section II presents the mathematical models of the snake robot used for simulation and stability analysis, respectively. The controller and the stability analysis are given in Section III. The simulation results are given in Section IV, and concluding remarks are found in Section V.

\section{MAthematical Modeling}

In this section, we first present the mathematical model of a planar snake robot without wheels based on the standard 'robot equation' with minimal coordinates [15]. We denote this model the process plant model and implement it for 


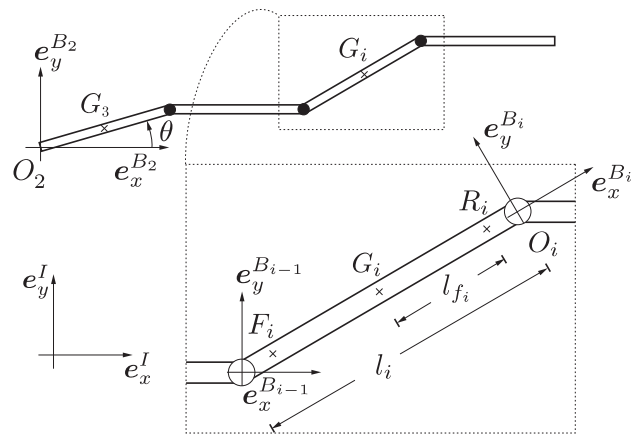

Fig. 1. Snake robot with 4 links. Notice that $G_{i}$ is the center of gravity of link $i=5$ in the picture.

two different constitutive laws for Coulomb friction. Subsequently, we will reformulate the process plant model to obtain a control plant model used both for implementing the joint controllers and for deriving stability results for the closed-loop system.

We include the derivation of models to ease the understanding of the forthcoming stability-proof. Moreover, the snake robot differs from a regular robot manipulator in that external forces act on each link instead of just the endeffector. This calls for a more extensive use of the various Jacobians.

\section{A. Process Plant Model}

In this section, we derive the mathematical model of a wheel-less snake robot based on the Euler-Lagrange equations as found in e.g. [15]. We present two models for the Coulomb friction between the snake robot and the ground. The first model is a smooth approximation to the Coulomb friction for simulation of a system that is equivalent to the control plant model. The second friction model is a model of set-valued Coulomb friction using a set-valued force law. We implement the latter model to check how the controller works for a more realistic model of a snake robot.

In the following we will first describe the kinematics and then we present the dynamics.

1) Kinematics: The 2D snake robot presented in this paper consists of $n-2$ equal links connected by $n-3$ revolute joints, each with one degree-of-freedom (DOF). See Fig. 1 for an illustration of a snake robot with four links. The earth-fixed position and orientation of the snake robot adds three DOF to the model so the snake robot has $n$ DOF. To be able to give a minimal coordinate representation of the snake robot in an earth-fixed frame, we need to include virtual joints in the model [16]. These joints give the position and orientation of the first link with respect to the earth-fixed frame and are not actual joints on the snake robot. Due to these virtual joints, we also obtain virtual links (link 1 and 2 ) in the model with no mass. Each (real) link $i=3, \ldots, n$ has length $l_{i}$ and the position of the center of gravity (CG) is given by the point $G_{i}$ as depicted in Fig. 1. We lump the multi-point contact between link $i$ and the ground into the two points, $F_{i}$ and $R_{i}$, each of distance $l_{f i}$ from $G_{i}$. We employ these two contact points to keep the model as simple as possible while still accounting for the friction forces that arise from a purely rotational motion of the link (this would not have been the case if $G_{i}$ was chosen as the contact point).

We denote the centre of the earth-fixed frame $I=$ $\left\{O_{I}, \boldsymbol{e}_{x}^{I}, \boldsymbol{e}_{y}^{I}\right\}$ as $O_{I}$. A general notation used throughout this paper is that a position vector from the origin of frame $I$ to a point $A$ is given by $\boldsymbol{r}_{A} \in \mathbb{R}^{2}$ and a vector from point A to point $\mathrm{B}$ is written $\boldsymbol{r}_{A B}$. Let a vector $\boldsymbol{r}_{A}$ described in frame $I$ be written as ${ }_{I} \boldsymbol{r}_{A}$. The body fixed frame $B_{i}=$ $\left\{O_{i}, \boldsymbol{e}_{x}^{B_{i}}, \boldsymbol{e}_{y}^{B i}\right\}$, for $i=3, \ldots, n$, is attached to the rear of link $i$ and is pointing along the link as shown in Fig. 1. The remaining frames $B_{i}, i=0, \ldots, 2$, are utilized to give the earth-fixed position and orientation of the first link of the snake robot and will be described shortly. The transformation of a vector $\boldsymbol{r}$ between frame $I$ and $B_{i}$ is given by

$$
{ }_{I} \boldsymbol{r}=\mathbf{R}_{B_{i} B_{i}}^{I} \boldsymbol{r},
$$

where $\mathbf{R}_{B_{i}}^{I} \in S O(2)$ is the corresponding rotation matrix.

We define $B_{0}=B_{1}=I$, and let ${ }_{I} \boldsymbol{r}_{O_{2}}=\left[\begin{array}{ll}x_{\mathrm{O}_{2}} & y_{\mathrm{O}_{2}}\end{array}\right]^{\mathrm{T}}$ be the position of the front part of the head of the snake robot (link 3) where $e_{x}^{B_{2}}=e_{x}^{I}$ and $e_{y}^{B_{2}}=e_{y}^{I}$. The orientation of link 3 is given by $\theta \in \mathcal{S}^{1}$. Let $\phi_{j} \in \mathcal{S}^{1}, j=1, \ldots, n-3$ be the relative joint angles between link $j+2$ and $j+3$, and define ${ }_{I} \boldsymbol{r}_{O_{0}}={ }_{I} \boldsymbol{r}_{O_{1}}={ }_{I} \boldsymbol{r}_{G_{1}}={ }_{I} \boldsymbol{r}_{G_{2}}=\left[\begin{array}{ll}0 & 0\end{array}\right]^{\mathrm{T}}$. Subsequently,

$$
\boldsymbol{r}_{O_{i}}=\boldsymbol{r}_{O_{i-1}}+l_{i} \boldsymbol{e}_{x}^{B_{i}}, \quad \boldsymbol{r}_{G_{i}}=\boldsymbol{r}_{O_{i}}-\frac{l_{i}}{2} \boldsymbol{e}_{x}^{B_{i}},
$$

for $i=3, \ldots, n$.

The generalized coordinates for the snake robot are

$$
\boldsymbol{q}=\left[\begin{array}{llllll}
x_{\mathrm{O}_{2}} & y_{\mathrm{O}_{2}} & \theta & \phi_{1} & \cdots & \phi_{n-3}
\end{array}\right]^{\mathrm{T}} \in \mathbb{R}^{n},
$$

where $\phi_{j}$ is the joint angle between link $j+2$ and $j+3$.

The mapping from the time-derivative $\dot{\boldsymbol{q}}$ of the generalized coordinates to the linear $\boldsymbol{v}$ and angular $\omega$ velocity of link $i$ is found with the manipulator Jacobian [15]. Next, we derive this Jacobian for the points $G_{i}$ in order obtain the mass matrix, and the Jacobian for $F_{i}$ and $R_{i}$ to be able to include friction in the system dynamics.

The translational velocity ${ }_{I} \boldsymbol{v}_{G_{i}}$ of the center of gravity of link $i=3, \ldots, n$ is found from

$$
{ }_{I} \boldsymbol{v}_{G_{i}}=\mathbf{J}_{v_{G i}}^{P}(\boldsymbol{q}) \dot{\boldsymbol{q}},
$$

where we used the superscript $P$ to denote the process plant model, and the Jacobian is found from

$\mathbf{J}_{v_{G i}}^{P}(\boldsymbol{q})=\left[\left(\boldsymbol{J}_{v_{G i}}^{P}\right)_{1}\left(\boldsymbol{J}_{v_{G i}}^{P}\right)_{2} \cdots\left(\boldsymbol{J}_{v_{G i}}^{P}\right)_{n}\right] \in \mathbb{R}^{2 \times n}$,

with

$\left(\boldsymbol{J}_{v_{G i}}^{P}\right) k= \begin{cases}{\left[\begin{array}{cc}0 & -1 \\ 1 & 0\end{array}\right]\left({ }_{I} \boldsymbol{r}_{G_{i}}-{ }_{I} \boldsymbol{r}_{O_{k-1}}\right)} & , \text { revolute joint } k \\ { }_{I} \boldsymbol{e}_{\zeta}^{B_{k-1}} & , \text { prismatic joint } k \\ {\left[\begin{array}{ll}0 & 0\end{array}\right]^{\mathrm{T}}} & , k>i,\end{cases}$

and $\zeta$ is the axis of elongation for the prismatic joint $k$. The rotational velocity $\omega_{i}$ of link $i=3, \ldots, n$ is

$$
\omega_{i}=\mathbf{J}_{\omega_{i}}^{P}(\boldsymbol{q}) \dot{\boldsymbol{q}}
$$

where $\mathbf{J}_{\omega_{i}}^{P}(\boldsymbol{q}) \in \mathbb{R}^{1 \times n}$ is constructed the same way as $\mathbf{J}_{v_{G i}}^{P}(\boldsymbol{q})$ in (5) for $\left(J_{\omega_{i}}^{P}\right)_{k}=1$ for a revolute joint $k$ and $\left(J_{\omega_{i}}^{P}\right)_{k}=0$ for a prismatic joint $k$ or $k>i$.

The above Jacobians are employed in the system dynamics to find the mass matrix. Since we have defined the friction to act on the links at positions different from the center of gravity, we also need to define an additional set of Jacobians. The translational velocities of $F_{i}$ and $R_{i}$ are found from

$$
{ }_{I} \boldsymbol{v}_{\xi_{i}}=\mathbf{J}_{v_{\xi i}}^{P}(\boldsymbol{q}) \dot{\boldsymbol{q}}
$$


for $\xi=F_{i}, R_{i}$ and $i=3, \ldots, n$, where $\mathbf{J}_{v_{\xi i}}^{P}(\boldsymbol{q}) \in \mathbb{R}^{2 \times n}$ has the same form as in (5) and $\left(\boldsymbol{J}_{v_{\xi i}}^{P}\right)_{k}$ is found the same way as $\left(\boldsymbol{J}_{v_{G i}}^{P}\right)_{k}$ by replacing ${ }_{I} \boldsymbol{r}_{G_{i}}$ with ${ }_{I} \boldsymbol{r}_{\xi_{i}}$ in (6), where $\boldsymbol{r}_{R_{i}}=\boldsymbol{r}_{G_{i}}+l_{f_{i}} \boldsymbol{e}_{x}^{B_{i}}$ and $\boldsymbol{r}_{F_{i}}=\boldsymbol{r}_{G_{i}}-l_{f_{i}} \boldsymbol{e}_{x}^{B_{i}}$.

2) Dynamics: The equations of motion for the snake robot are based on the Euler-Lagrange equations and is formulated in a standard matrix form for planar robot manipulators (see e.g. [15]) as follows:

$$
\begin{aligned}
& \mathbf{M}_{P}(\boldsymbol{q}) \ddot{\boldsymbol{q}}+\mathbf{C}_{P}(\boldsymbol{q}, \dot{\boldsymbol{q}}) \dot{\boldsymbol{q}}=\mathbf{P}_{\phi} \boldsymbol{\tau}+ \\
& \sum_{i=3}^{n}\left(\left(\mathbf{J}_{v_{F i}}^{P}\right)^{\mathrm{T}}{ }_{I} \boldsymbol{F}_{F_{i}}^{P}+\left(\mathbf{J}_{v_{R i}}^{P}\right)^{\mathrm{T}}{ }_{I} \boldsymbol{F}_{R_{i}}^{P}\right) \text {, }
\end{aligned}
$$

where the mass matrix $\mathbf{M}_{P}(\boldsymbol{q}) \in \mathbb{R}^{n \times n}$, Coriolis and centripetal matrix $\mathbf{C}_{P}(\boldsymbol{q}, \dot{\boldsymbol{q}}) \in \mathbb{R}^{n \times n}$, the applied joint torques $\mathbf{P}_{\phi} \boldsymbol{\tau}$, and the friction forces ${ }_{I} \boldsymbol{F}_{F_{i}}^{P}$ and ${ }_{I} \boldsymbol{F}_{R_{i}}^{P}$ will be described in the following paragraphs.

The mass matrix is

$$
\mathbf{M}_{P}(\boldsymbol{q})=\sum_{i=3}^{n}\left(m_{i}\left(\mathbf{J}_{v_{G i}}^{P}\right)^{\mathrm{T}} \mathbf{J}_{v_{G i}}^{P}+\Theta_{i}\left(\mathbf{J}_{\omega_{i}}^{P}\right)^{\mathrm{T}} \mathbf{J}_{\omega_{i}}^{P}\right)
$$

where $m_{i}$ and $\Theta_{i}$ are the mass and moment of inertia of link $i$ with respect to its center of mass, respectively $\left(m_{1}=m_{2}=\right.$ $\Theta_{1}=\Theta_{2}=0$ due to the virtual joints and are therefore not included in $\mathbf{M}_{P}(\boldsymbol{q})$ ). By defining $\mathbf{M}_{P}(\boldsymbol{q})=\left\{M_{P_{i j}}\right\}$ and $\mathbf{C}_{P}(\boldsymbol{q}, \dot{\boldsymbol{q}})=\left\{C_{P_{i j}}\right\}$ we can find the elements of the Coriolis and centripetal matrix as

$$
C_{P_{i j}}=\sum_{k=1}^{n} c_{i j k} \dot{q}_{k}
$$

where

$$
c_{i j k}=\frac{1}{2}\left(\frac{\partial M_{P_{i j}}}{\partial q_{k}}+\frac{\partial M_{P_{i k}}}{\partial q_{j}}-\frac{\partial M_{P_{k j}}}{\partial q_{i}}\right) .
$$

The torques $\tau \in \mathbb{R}^{n-3}$ applied by the motors in the joints are mapped into the system dynamics by the constant matrix

$$
\mathbf{P}_{\phi}=\left[\begin{array}{c}
\mathbf{0}_{3 \times n-3} \\
\mathbf{I}_{n-3 \times n-3}
\end{array}\right],
$$

where $\mathbf{I}_{n-3 \times n-3}$ is the $n-3 \times n-3$ identity matrix. The friction between the ground surface and the snake robot links is modeled as a combination of Coulomb friction $\boldsymbol{F}_{F_{i}}^{P_{C}}, \boldsymbol{F}_{R_{i}}^{P_{C}}$, and viscous friction $\boldsymbol{F}_{F_{i}}^{P_{V}}, \boldsymbol{F}_{R_{i}}^{P_{V}}$. Hence, we have

$$
\boldsymbol{F}_{\xi_{i}}^{P}=\boldsymbol{F}_{\xi_{i}}^{P_{C}}+\boldsymbol{F}_{\xi_{i}}^{P_{V}},
$$

for $\xi_{i}=F_{i}, R_{i}$. We formulate the Coulomb friction force law in two ways. First we present a smooth approximation to Coulomb friction $\boldsymbol{F}_{\xi_{i}}^{P_{C_{c}}}$, and then we show how to find the set-valued Coulomb friction $\boldsymbol{F}_{\xi_{i}}^{P_{C_{t}}}$.

Coulumb friction is often modeled with a sign-function [5], [17], and the arctan-function can be employed as an approximation to the sign-function. Hence, we can find a smooth approximation of the Coulomb friction $\boldsymbol{F}_{\xi_{i}}^{P_{C_{c}}}$ from

$$
{ }_{B_{i}} \boldsymbol{F}_{\xi_{i}}^{P_{C_{c}}}=-F_{N_{i}} \mathbf{D}_{\mu_{C}} \frac{1}{\pi} \arctan \left(\frac{B_{i} \boldsymbol{v}_{\xi_{i}}}{\epsilon}\right)
$$

where $F_{N_{i}}=m_{i} g, g$ is the acceleration of gravity, $\epsilon>0$ is a small constant, the diagonal matrix $\mathbf{D}_{\mu_{C}}=$ $\operatorname{diag}\left(\left[\mu_{C_{x}}, \mu_{C_{y}}\right]\right)$, and $\mu_{C_{x}} \in \mathbb{R}^{+}, \mu_{C_{y}} \in \mathbb{R}^{+}$are the Coulomb friction coefficients longitudinal and lateral to each link, respectively. Notice that the normal force $F_{N_{i}}$ is divided equally between the two contact points on each link. In addition, we employ the common assumption that the normal forces are equally distributed along the snake robot [5], [6].

Set-valued Coulomb friction $\boldsymbol{F}_{\xi_{i}}^{P_{C_{t}}}$ is modeled using a set-valued force law based on the framework of non-smooth dynamics and convex analysis, and an expression for the friction is given with the algebraic inclusion

$$
-{ }_{B_{i}} \boldsymbol{v}_{\xi_{i}} \in N_{C_{T}}\left({ }_{B} \boldsymbol{F}_{\xi_{i}}^{P_{C_{t}}}\right),
$$

where $N_{C_{T}}(\cdot)$ is the normal cone to the set $C_{T}$ given by the ellipse:

$$
C_{T}=\left\{B_{i} \boldsymbol{F}_{\xi_{i}}^{P_{C_{t}}} \mid \frac{\left(B_{i} F_{\xi_{i x}}^{P_{C_{t}}}\right)^{2}}{\left(\mu_{C_{x}} \frac{F_{N_{i}}}{2}\right)^{2}}+\frac{\left(B_{i} F_{\xi_{i y}}^{P_{C_{t}}}\right)^{2}}{\left(\mu_{C_{y}} \frac{F_{N_{i}}}{2}\right)^{2}} \leq 1\right\}
$$

where we have defined ${ }_{B} \boldsymbol{F}_{\xi_{i}}^{P_{C_{t}}}=\left[\begin{array}{ll}B_{i} F_{\xi_{i x}}^{P_{C_{t}}} & { }_{B} F_{\xi_{i y}}^{P_{C_{t}}}\end{array}\right]^{\mathrm{T}}$ (see [10], [11], [13], [18], [19] for an introduction to setvalued force laws and non-smooth dynamics).

The viscous friction force can be expressed in frame $B_{i}$ as

$$
{ }_{B_{i}} \boldsymbol{F}_{\xi_{i}}^{P_{V}}=-\frac{F_{N_{i}}}{2} \mathbf{D}_{\mu_{V} B_{i}} \boldsymbol{v}_{\xi_{i}}, \quad \mathbf{D}_{\mu_{V}}=\left[\begin{array}{cc}
\mu_{V_{x}} & 0 \\
0 & \mu_{V_{y}}
\end{array}\right]
$$

where $\mu_{V_{x}} \in \mathbb{R}^{+}$and $\mu_{V_{y}} \in \mathbb{R}^{+}$are the viscous friction coefficients longitudinal and lateral to each link, respectively.

\section{B. Control Plant Model}

The control plant model is derived from the process plant model in this section. We approximate the set-valued Coulomb friction law (16) by the smooth nonlinear friction model (15). This enables us to apply input-output linearization techniques developed for smooth systems. We will revert to the performance of the controller for the non-smooth system in Section IV. We reformulate the model such that the position ${ }_{I} \boldsymbol{r}_{\mathrm{O}_{2}}=\left[\begin{array}{ll}x_{\mathrm{O}_{2}} & y_{\mathrm{O}_{2}}\end{array}\right]^{\mathrm{T}}$ and orientation $\theta$ are removed from the state equations in (9).

1) Kinematics: In this section, we present the new kinematics where the earth-fixed position and orientation of the snake robot have been decoupled from the rest of the system dynamics. To achieve this, we define a new vector $\nu$ of velocity coordinates that includes the velocity ${ }_{I} \boldsymbol{v}_{O_{2}}={ }_{I} \dot{\boldsymbol{r}}_{O_{2}}$ in frame $B_{3}$ attached to the first link: ${ }_{B_{3}} \boldsymbol{v}_{O_{2}}=\left(R_{B_{3}}^{I}\right)^{\mathrm{T}}{ }_{I} \boldsymbol{v}_{O_{2}}$. Hence,

$$
\dot{\boldsymbol{q}}=\mathbf{T}_{q} \boldsymbol{\nu} \Longleftrightarrow \boldsymbol{\nu}=\mathbf{T}_{q}^{\mathrm{T}} \dot{\boldsymbol{q}}
$$

where

$$
\begin{aligned}
& \mathbf{T}_{q}=\mathbf{T}_{q}(\theta)=\left[\begin{array}{cc}
\mathbf{R}_{B_{3}}^{I} & \mathbf{0}_{2 \times n-2} \\
\mathbf{0}_{n-2 \times 2} & \mathbf{I}_{n-2 \times n-2}
\end{array}\right] \\
& \boldsymbol{\nu}=\left[{ }_{B_{3}} \boldsymbol{v}_{O_{2}}^{\mathrm{T}} \dot{\theta} \dot{\boldsymbol{\phi}}^{\mathrm{T}}\right]^{\mathrm{T}}, \boldsymbol{\phi}^{\mathrm{T}}=\left[\begin{array}{llll}
\phi_{1} & \phi_{2} & \cdots & \phi_{n-3}
\end{array}\right] .
\end{aligned}
$$

To be able to cancel $\theta$ from the model, we describe all translational velocities in frame $B_{3}$. Let us investigate the new expression for the translational velocity $\boldsymbol{v}_{F_{i}}$ of the front part of link $i$ as an example: By inserting (19) into (8) we find that

where

$$
{ }_{B 3} \boldsymbol{v}_{F_{i}}=\mathbf{J}_{v_{F i}}(\phi) \boldsymbol{\nu}
$$

$$
\mathbf{J}_{v_{F i}}(\phi)=\left(\mathbf{R}_{B_{3}}^{I}\right)^{\mathrm{T}} \mathbf{J}_{v_{F i}}^{P} \mathbf{T}_{q}
$$

The expression for ${ }_{B_{3}} \boldsymbol{v}_{R_{i}}$ is the same as (22) except that $F_{i}$ needs to be replaced by $R_{i}$. The Jacobian $\mathbf{J}_{v_{F i}}(\phi)$ that relates the new generalized velocities $\nu$ and the translational 
velocity ${ }_{B_{3}} \boldsymbol{v}_{F_{i}}$ does not depend on the earth-fixed rotation angle $\theta$ of the snake robot head (link 3). Namely, the translational velocities of the snake robot head in $\nu$ are given in frame $B_{3}$ and an earth-fixed rotation angle is therefore no longer necessary to find the translational link velocities in frame $B_{3}$.

2) Dynamics: In this section, we employ the transformations above to reduce the model of the dynamics (9). From (19), we find that

$$
\ddot{\boldsymbol{q}}=\dot{\mathbf{T}}_{q} \boldsymbol{\nu}+\mathbf{T}_{q} \dot{\boldsymbol{\nu}},
$$

and this equality together with (19) and the relation ${ }_{I} \boldsymbol{F}_{\xi_{i}}^{P}=$ $\mathbf{R}_{B_{3} \quad B_{3}}^{I} \boldsymbol{F}_{\xi_{i}}^{P}$ are inserted into (9) to obtain

$$
\begin{gathered}
\mathbf{M}_{P}(\boldsymbol{q}) \mathbf{T}_{q} \dot{\boldsymbol{\nu}}+\left(\mathbf{M}_{P}(\boldsymbol{q}) \dot{\mathbf{T}}_{q}+\mathbf{C}_{P}(\boldsymbol{q}, \dot{\boldsymbol{q}}) \mathbf{T}_{q}\right) \boldsymbol{\nu}= \\
\mathbf{P}_{\phi} \boldsymbol{\tau}+\sum_{i=3}^{n}\left(\left(\mathbf{J}_{v_{F i}}^{P}\right)^{\mathrm{T}} \mathbf{R}_{B_{3} B_{3}}^{I} \boldsymbol{F}_{F_{i}}^{P}+\left(\mathbf{J}_{v_{R i}}^{P}\right)^{\mathrm{T}} \mathbf{R}_{B_{3} B_{3}}^{I} \boldsymbol{F}_{R_{i}}^{P}\right) .
\end{gathered}
$$

We pre-multiply (25) with $\mathbf{T}_{q}^{\mathrm{T}}$ and using (23) and $\mathbf{T}_{q}^{\mathrm{T}} \mathbf{P}_{\phi}=$ $\mathbf{P}_{\phi}$, we obtain

$$
\begin{gathered}
\mathbf{M}(\boldsymbol{\phi}) \dot{\boldsymbol{\nu}}+\mathbf{C}(\dot{\theta}, \boldsymbol{\phi}, \dot{\boldsymbol{\phi}}) \boldsymbol{\nu}= \\
\mathbf{P}_{\phi} \boldsymbol{\tau}+\sum_{i=3}^{n}\left(\left(\mathbf{J}_{v_{F i}}\right)^{\mathrm{T}}{ }_{B_{3}} \boldsymbol{F}_{F_{i}}+\left(\mathbf{J}_{v_{R i}}\right)^{\mathrm{T}}{ }_{B_{3}} \boldsymbol{F}_{R_{i}}\right) .
\end{gathered}
$$

where

$$
\begin{gathered}
\mathbf{M}(\boldsymbol{\phi})=\mathbf{T}_{q}^{\mathrm{T}} \mathbf{M}_{P}(\boldsymbol{q}) \mathbf{T}_{q} \\
\mathbf{C}(\dot{\theta}, \boldsymbol{\phi}, \dot{\boldsymbol{\phi}})=\mathbf{T}_{q}^{\mathrm{T}}\left(\mathbf{M}_{P}(\boldsymbol{q}) \dot{\mathbf{T}}_{q}+\mathbf{C}_{P}(\boldsymbol{q}, \dot{\boldsymbol{q}}) \mathbf{T}_{q}\right),
\end{gathered}
$$

and we have used that $\boldsymbol{F}_{\xi_{i}}=\boldsymbol{F}_{\xi_{i}}^{C}+\boldsymbol{F}_{\xi_{i}}^{V}=\boldsymbol{F}_{\xi_{i}}^{P}$ with $\xi_{i}=F_{i}, R_{i}$ where the viscous friction term $\boldsymbol{F}_{\xi_{i}}^{V}=\boldsymbol{F}_{\xi_{i}}^{P_{V}}$ is the same as in (18), while the smooth approximation $\boldsymbol{F}_{\xi_{i}}^{C}=$ $\boldsymbol{F}_{\xi_{i}}^{P_{C_{c}}}$ in (15) of the Coulomb friction in the process plant model is employed.

\section{JOINT CONTROL BY INPUT-OUTPUT LINEARIZATION}

In this section, we employ an input-output (IO) linearizing controller to be able to accurately track the desired joint angles and prove mathematically that this closed-loop system is stable. Inspired by [20], we divide the system (26) into two parts: one part for the directly actuated generalized coordinates, called active (or real) joints, and one part for the unactuated degrees of freedom, the passive (or virtual) joints. We will then show that the origin of the tracking error dynamics of the active joints are asymptotically stable and that the IO-linearizing controller can be employed.

\section{A. Control Plant Model Reformulation}

We begin by dividing the control plant model (26) into two parts (as performed for the standard robotic equation in [20]): one part for the directly actuated generalized coordinates $\phi$ called active joints, and one part for the unactuated generalized velocities $\boldsymbol{w}$, the passive joints. We define the unactuated generalized velocities as

$$
\boldsymbol{w}=\left[\begin{array}{c}
B_{3} \boldsymbol{v}_{O_{2}} \\
\dot{\theta}
\end{array}\right] \in \mathbb{R}^{3} .
$$

Define

$$
\begin{aligned}
\boldsymbol{h} & =\mathbf{C}(\dot{\theta}, \boldsymbol{\phi}, \dot{\boldsymbol{\phi}}) \boldsymbol{\nu} \\
\boldsymbol{g} & =\sum_{i=3}^{n}\left(\left(\mathbf{J}_{v_{F i}}\right)^{\mathrm{T}}{ }_{B_{3}} \boldsymbol{F}_{F_{i}}+\left(\mathbf{J}_{v_{R i}}\right)^{\mathrm{T}}{ }_{B_{3}} \boldsymbol{F}_{R_{i}}\right),
\end{aligned}
$$

where $\boldsymbol{h}(\boldsymbol{\phi}, \boldsymbol{\nu})=\left[\begin{array}{ll}\boldsymbol{h}_{1}^{\mathrm{T}} & \boldsymbol{h}_{2}^{\mathrm{T}}\end{array}\right]^{\mathrm{T}}, \boldsymbol{g}(\boldsymbol{\phi}, \boldsymbol{\nu})=\left[\begin{array}{ll}\boldsymbol{g}_{1}^{\mathrm{T}} & \boldsymbol{g}_{2}^{\mathrm{T}}\end{array}\right]^{\mathrm{T}}$, $\boldsymbol{h}_{1} \in \mathbb{R}^{3}, \boldsymbol{h}_{2} \in \mathbb{R}^{n-3}, \boldsymbol{g}_{1} \in \mathbb{R}^{3}$, and $\boldsymbol{g}_{2} \in \mathbb{R}^{n-3}$. Then, the control plant model may be written as follows:

$$
\begin{aligned}
& \mathbf{M}_{11} \dot{\boldsymbol{w}}+\mathrm{M}_{12} \ddot{\boldsymbol{\phi}}+\boldsymbol{h}_{1}=\boldsymbol{g}_{1} \\
& \mathrm{M}_{21} \dot{\boldsymbol{w}}+\mathrm{M}_{22} \ddot{\boldsymbol{\phi}}+\boldsymbol{h}_{2}=\boldsymbol{g}_{2}+\boldsymbol{\tau},
\end{aligned}
$$

where

$$
\mathbf{M}(\phi)=\left[\begin{array}{ll}
\mathbf{M}_{11} & \mathbf{M}_{12} \\
\mathbf{M}_{21} & \mathbf{M}_{22}
\end{array}\right], \quad \mathbf{M}_{11} \in \mathbb{R}^{3 \times 3} .
$$

In the forthcoming section, we denote (32) as the tracking dynamics and (33) as the input-output dynamics.

\section{B. Controller Design}

In this section, we derive an input-output linearizing controller such that the system (33) is able to asymptotically track a bounded reference $\phi_{d}(t)$, with bounded derivatives $\dot{\phi}_{d}(t), \ddot{\phi}_{d}(t)$.

Since $\mathbf{M}(\phi)$ is positive definite, then $\mathbf{M}_{11}$ is invertible and we may substitute $\dot{\boldsymbol{w}}$ from (32) into (33) to obtain

$$
\overline{\mathbf{M}}_{22} \ddot{\boldsymbol{\phi}}+\overline{\boldsymbol{h}}_{2}=\overline{\boldsymbol{g}}_{2}+\boldsymbol{\tau}
$$

where

$$
\begin{aligned}
\overline{\mathbf{M}}_{22} & =\mathbf{M}_{22}-\mathbf{M}_{21} \mathbf{M}_{11}^{-1} \mathbf{M}_{12} \\
\overline{\boldsymbol{h}}_{2} & =\boldsymbol{h}_{2}-\mathbf{M}_{21} \mathbf{M}_{11}^{-1} \boldsymbol{h}_{1} \\
\overline{\boldsymbol{g}}_{2} & =\boldsymbol{g}_{2}-\mathbf{M}_{21} \mathbf{M}_{11}^{-1} \boldsymbol{g}_{1},
\end{aligned}
$$

Note that $\overline{\mathbf{M}}_{22}$ is symmetric and positive definite [21]. The friction and system dynamics may now be cancelled from (35) by employing the feedback transformation

$$
\boldsymbol{\tau}=\overline{\mathbf{M}}_{22} \boldsymbol{u}+\overline{\boldsymbol{h}}_{2}-\overline{\boldsymbol{g}}_{2}
$$

where $\boldsymbol{u} \in \mathbb{R}^{n-3}$ is a new control input. The input-output dynamics (33) is now reduced to

$$
\ddot{\phi}=u \text {. }
$$

Hence, by using the stabilizing feedback law for $\boldsymbol{u} \in \mathbb{R}^{n-3}$

$$
\boldsymbol{u}=\ddot{\boldsymbol{\phi}}_{d}-\mathbf{K}_{D}\left(\dot{\boldsymbol{\phi}}-\dot{\boldsymbol{\phi}}_{d}\right)-\mathbf{K}_{P}\left(\boldsymbol{\phi}-\boldsymbol{\phi}_{d}\right),
$$

we obtain an asymtotically stable error dynamics for the system (33) for the $n-3 \times n-3$ positive definite, diagonal, and constant matrices $\mathbf{K}_{D}$ and $\mathbf{K}_{P}$. We see from (31) that $\overline{\boldsymbol{g}}_{2}$ in the controller (39) contains the Coulomb and viscous friction terms. Now, the reason for the approximation (15) of the Coulomb friction becomes apparent. Namely, due to the set-valued Coulomb friction in the process plant model we are not able to compensate exactly for the Coulomb friction at zero velocity since the friction force can be anywhere in the set $C_{T}$ in (17). To be able to include the case of zero velocity in the stability proof we therefore approximate the Coulomb friction with the smooth friction model as in (15).

\section{Motion Pattern and Reference Angles}

The 'serpenoid curve' is a sinus-like curve that describes the shape of a snake during the serpenoid motion pattern 'lateral undulation' [2]. A snake robot may recreate an approximation of this shape by setting its joint angles as

$$
\phi_{d_{i}}(t)=A \sin (\omega t+(i-1) \delta),
$$

where $\phi_{d_{i}}(t)$ is the desired relative angle between link $i+2$ and $i+3, A$ is the amplitude of joint oscillation, $\omega$ is the speed of the propagating wave, $t$ is the time, and $\delta$ is the 
phase-shift between adjacent joints [5]. It has been shown that the snake robot in fact moves forward when its joint angles are set to follow $\phi_{d_{i}}$ and the friction property between each link of the snake robot and the ground is such that a link glides easier forward and backwards than sideways [2], [5], [22], [23]. In this paper we employ (42) to find $\phi_{d}$ in (41).

\section{Final Results}

We start this section by stating the following theorem.

Theorem 1: Assume that the desired trajectory $\phi_{d}$ is bounded with bounded velocity $\dot{\phi}_{d}$ and acceleration $\ddot{\phi}_{d}$. Along any trajectory of the closed-loop (32)-(33), (39), (41), the joint tracking error $\boldsymbol{e}_{\phi}(t)$, defined by

$$
\boldsymbol{e}_{\phi}(t)=\phi(t)-\phi_{d}(t)
$$

converges to zero exponentially fast, and the velocity vector $\boldsymbol{w}$ remains bounded.

Proof: The desired trajectory $\phi_{d}$, together with its timederivatives, typically satisfies the boundedness assumption (e.g. by employing (42) for bounded $A, \omega$, and $\delta$ ).

The convergence to zero of the joint tracking error $\boldsymbol{e}_{\phi}(t)$ is easily seen from (40)-(41). Hence, it now remains to show that the velocity vector $\boldsymbol{w}$ that appears in the closed-loop (32)-(33), (39), (41), and that was removed from (33) using (32), does not grow unbounded, i.e. the so-called tracking dynamics (32) produces bounded solutions for time-varying inputs $\phi_{d}(t), \dot{\phi}_{d}(t)$, and $\ddot{\phi}_{d}(t)$.

Notice that, due to (43),

$$
\phi(t)=\boldsymbol{e}_{\phi}(t)+\phi_{d}(t) .
$$

Hence, $\phi(t)$ is bounded since both $e_{\phi}(t)$ and $\phi_{d}(t)$ are bounded. The same applies to the first and second derivative of $\phi(t)$. For notational simplicity, we omit the dependence of $t$ and $\phi(t)$ in the following when it is appropriate. We now want to show that $\boldsymbol{w}$ is bounded. To this end, we employ Theorem 4.18 in [24] and define the continuously differentiable function

Thus,

$$
V(t, \boldsymbol{w})=\frac{1}{2} \boldsymbol{w}^{\mathrm{T}} \mathbf{M}_{11}(\boldsymbol{\phi}(t)) \boldsymbol{w} .
$$

$$
\frac{1}{2} \lambda_{M, \min }\|\boldsymbol{w}\|^{2} \leq V(t, \boldsymbol{w}) \leq \frac{1}{2} \lambda_{M, \max }\|\boldsymbol{w}\|^{2}
$$

where $\|\cdot\|$ denotes the Euclidean norm, $\lambda_{M, \text { min }}$ is the infimum over all $t$ of the smallest eigenvalue of $\mathbf{M}_{11}(\phi(t))$, and $\lambda_{M, \max }$ is the supremum over all $t$ of the largest eigenvalue of $\mathbf{M}_{11}(\phi(t))$. Since $\mathbf{M}_{11}(\phi)$ is continuous and positive definite for all $\phi$, and since $\phi(t)$ belongs to a compact set for all $t$, then $\lambda_{M, \max } \geq \lambda_{M, \min }>0$. What remains is to find a continuous positive definite function $W(\boldsymbol{w})$ such that

$$
\dot{V}(t, \boldsymbol{w}) \leq-W(\boldsymbol{w}), \quad \forall\|\boldsymbol{w}\| \geq \mu>0, \quad \forall t \geq 0,
$$

and $\forall \boldsymbol{w} \in \mathbb{R}^{3}$. This will be the task for the remainder of this section. Consider

$$
\dot{V}=\boldsymbol{w}^{\mathrm{T}} \mathbf{M}_{11} \dot{\boldsymbol{w}}+\frac{1}{2} \boldsymbol{w}^{\mathrm{T}} \dot{\mathbf{M}}_{11} \boldsymbol{w}
$$

Using (32), we find that

$$
\dot{V}=\boldsymbol{w}^{\mathrm{T}}\left(\boldsymbol{g}_{1}-\mathbf{M}_{12} \ddot{\boldsymbol{\phi}}-\boldsymbol{h}_{1}\right)+\frac{1}{2} \boldsymbol{w}^{\mathrm{T}} \dot{\mathbf{M}}_{11} \boldsymbol{w} .
$$

The velocities $\boldsymbol{w}$ need to be extracted from $\boldsymbol{g}_{1}$ and $\boldsymbol{h}_{1}$ to satisfy the inequality (47). Define

$$
\mathbf{P}_{w}=\left[\begin{array}{c}
\mathbf{I}_{3 \times 3} \\
\mathbf{0}_{n-3 \times 3}
\end{array}\right] \text {. }
$$

Hence, from (21), we have that

$$
\boldsymbol{\nu}=\mathbf{P}_{w} \boldsymbol{w}+\mathbf{P}_{\phi} \dot{\boldsymbol{\phi}} .
$$

where $\mathbf{P}_{\phi}$ is found in (13).

We start by investigating $\boldsymbol{h}_{1}$ which is the term in $\dot{V}$ that needs to be elaborated the most to prove the inequality (47). From (30), we find by employing the identity (51) that

$$
\boldsymbol{h}_{1}=\mathbf{P}_{w}^{\mathrm{T}} \mathbf{C}(\dot{\theta}, \boldsymbol{\phi}, \dot{\boldsymbol{\phi}}) \mathbf{P}_{w} \boldsymbol{w}+\mathbf{P}_{w}^{\mathrm{T}} \mathbf{C}(\dot{\theta}, \boldsymbol{\phi}, \dot{\boldsymbol{\phi}}) \mathbf{P}_{\phi} \dot{\boldsymbol{\phi}} .
$$

It will later be shown that the term $\mathbf{P}_{w}^{\mathrm{T}} \mathbf{C}(\dot{\theta}, \boldsymbol{\phi}, \dot{\boldsymbol{\phi}}) \mathbf{P}_{w} \boldsymbol{w}$ is cancelled from $\dot{V}$ because of skew-symmetry, however $\dot{\theta}$, and thus an expression for $\boldsymbol{w}$, needs to be extracted from the second term in $\boldsymbol{h}_{1}$ and this will be elaborated on in the following. By defining $\mathbf{C}_{1}=\mathbf{P}_{w}^{\mathrm{T}} \mathbf{C}$ and using $\dot{\mathbf{T}}_{q} \mathbf{P}_{\phi}=\mathbf{0}$ $\left(\dot{\mathbf{I}}_{n-2 \times n-2}=0\right)$ together with the transformation (28) we obtain

$$
\mathbf{C}_{1} \mathbf{P}_{\phi}=\mathbf{P}_{w}^{\mathrm{T}} \mathbf{T}_{q}^{\mathrm{T}} \mathbf{C}_{P} \mathbf{P}_{\phi},
$$

where we also have used that $\mathbf{T}_{q} \mathbf{P}_{\phi}=\mathbf{P}_{\phi}$. Define $\boldsymbol{c}_{j}$ to be the $j$-th column of $\mathbf{C}_{P}$ and $\boldsymbol{p}_{j}^{\mathrm{T}}=\left[\begin{array}{lll}\mathbf{0}_{1 \times j-1} & 1 & \mathbf{0}_{1 \times n-j}\end{array}\right] \in \mathbb{R}^{n}$ is a row vector of zeros with 1 on its $j$-th column. From the expression for the elements of $\mathbf{C}_{P}$ in (11) we define

$$
\boldsymbol{c}_{j}=\mathbf{D}_{P_{j}} \dot{\boldsymbol{q}}
$$

where $\mathbf{D}_{P_{j}}=\left\{c_{i j k}\right\}$, where $i$ denotes the row and $k$ the column, and $c_{i j k}$ is given by (12). By calculating the partial derivatives of the elements of $\mathbf{M}_{P}$ in (12) we find from the expression for the elements of $\mathbf{D}_{P_{j}}$ that its first two columns are zero. Hence, by comparing $\dot{\boldsymbol{q}}$ in (3) with $\boldsymbol{\nu}$ in (21) we obtain

$$
\mathrm{D}_{P_{j}} \dot{\boldsymbol{q}}=\mathrm{D}_{P_{j}} \boldsymbol{\nu} .
$$

From (11), (51), (54), and (55) we write $\mathbf{C}_{P}$ as

$$
\mathbf{C}_{P}=\sum_{j=1}^{n} \boldsymbol{c}_{j} \boldsymbol{p}_{j}^{\mathrm{T}}=\sum_{j=1}^{n} \mathbf{D}_{P_{j}}\left(\mathbf{P}_{w} \boldsymbol{w}+\mathbf{P}_{\phi} \dot{\boldsymbol{\phi}}\right) \boldsymbol{p}_{j}^{\mathrm{T}}
$$

By inserting (56) into (53) and noticing that $\boldsymbol{p}_{j}^{\mathrm{T}} \mathbf{P}_{\phi}=\mathbf{0}_{1 \times n-3}$ for $j=1, \ldots, 3$ we obtain

$$
\mathbf{C}_{1} \mathbf{P}_{\phi}=\mathbf{P}_{w}^{\mathrm{T}} \mathbf{T}_{q}^{\mathrm{T}} \sum_{j=4}^{n}\left\{\mathbf{D}_{P_{j}} \mathbf{P}_{w} \boldsymbol{w}+\mathbf{D}_{P_{j}} \mathbf{P}_{\phi} \dot{\boldsymbol{\phi}}\right\} \boldsymbol{p}_{j}^{\mathrm{T}} \mathbf{P}_{\phi}
$$

Inspecting the terms with $\boldsymbol{w}$ in the summation (57) for $j=$ $4, \ldots, n$ we find that

$$
\boldsymbol{p}_{\phi_{j-3}}^{\mathrm{T}}=\boldsymbol{p}_{j}^{\mathrm{T}} \mathbf{P}_{\phi}
$$

where $\boldsymbol{p}_{\phi_{j-3}}^{\mathrm{T}}=\left[\begin{array}{lll}\mathbf{0}_{1 \times j-3-1} & 1 & \mathbf{0}_{1 \times n-j}\end{array}\right] \in \mathbb{R}^{n-3}$ is a row vector of zeros with 1 on its $j-3$-column, and we note that

$$
\dot{\phi}_{k}=\boldsymbol{p}_{\phi_{k}}^{\mathrm{T}} \dot{\boldsymbol{\phi}}
$$

where $\phi_{k}$ is the $k$-th element of $\phi \in \mathbb{R}^{n-3}$. In addition, let

$$
\mathbf{D}_{B_{j}}(\phi)=\mathbf{T}_{q}^{\mathrm{T}} \mathbf{D}_{P_{j}} \in \mathbb{R}^{n \times n}, \quad j=4, \ldots, n,
$$

to explicitly show that we are dealing with a function only of $\phi$. By inserting (58) into (57), and employing the identities (59) and (60), we now find the second term of $\boldsymbol{h}_{1}$ in (52) as

$$
\mathbf{P}_{w}^{\mathrm{T}} \mathbf{C P}_{\phi} \dot{\boldsymbol{\phi}}=\mathbf{P}_{w}^{\mathrm{T}} \sum_{j=4}^{n} \mathbf{D}_{B_{j}}\left(\mathbf{P}_{\phi} \dot{\boldsymbol{\phi}} \dot{\phi}_{j-3}+\mathbf{P}_{w} \dot{\phi}_{j-3} \boldsymbol{w}\right)
$$


and we have managed to extract $\boldsymbol{w}$.

We are now ready to insert $\boldsymbol{h}_{1}$ into $\dot{V}$ in (49):

$$
\begin{aligned}
\dot{V}= & \boldsymbol{w}^{\mathrm{T}} \boldsymbol{g}_{1}+\boldsymbol{w}^{\mathrm{T}}\left(-\mathbf{M}_{12}\right) \ddot{\boldsymbol{\phi}}+\frac{1}{2} \boldsymbol{w}^{\mathrm{T}} \dot{\mathbf{M}}_{11} \boldsymbol{w}+ \\
& \boldsymbol{w}^{\mathrm{T}} \mathbf{P}_{w}^{\mathrm{T}}(-\mathbf{C}) \mathbf{P}_{w} \boldsymbol{w}+ \\
& \boldsymbol{w}^{\mathrm{T}} \mathbf{P}_{w}^{\mathrm{T}} \sum_{j=4}^{n}\left(-\mathbf{D}_{B_{j}}\right) \mathbf{P}_{\phi} \dot{\boldsymbol{\phi}} \dot{\phi}_{j-3}+ \\
& \boldsymbol{w}^{\mathrm{T}} \mathbf{P}_{w}^{\mathrm{T}} \sum_{j=4}^{n}\left(-\mathbf{D}_{B_{j}}\right) \mathbf{P}_{w} \dot{\phi}_{j-3} \boldsymbol{w} .
\end{aligned}
$$

By defining

$$
\begin{aligned}
& \boldsymbol{k}_{1}(\boldsymbol{\phi}, \ddot{\boldsymbol{\phi}})=\left(-\mathbf{M}_{12}\right) \ddot{\boldsymbol{\phi}} \\
& \boldsymbol{k}_{2}(\boldsymbol{\phi}, \dot{\boldsymbol{\phi}})=\mathbf{P}_{w}^{\mathrm{T}} \sum_{j=4}^{n}\left(-\mathbf{D}_{B_{j}}\right) \mathbf{P}_{\phi} \dot{\boldsymbol{\phi}} \dot{\phi}_{j-3},
\end{aligned}
$$

employing the inequality

$$
\boldsymbol{w}^{\mathrm{T}} \boldsymbol{k}_{i} \leq\|\boldsymbol{w}\|\left\|\boldsymbol{k}_{i}\right\|
$$

noting that

$$
\boldsymbol{w}^{\mathrm{T}} \mathbf{P}_{w}^{\mathrm{T}}\left(-\mathbf{D}_{B_{j}}(\phi(t))\right) \mathbf{P}_{w} \dot{\phi}_{j-3}(t) \boldsymbol{w} \leq \lambda_{D_{B j}, \max }\|\boldsymbol{w}\|^{2},
$$

where $\lambda_{D_{B j}, \max }$ is the supremum for all $t$ of the largest eigenvalue of $\mathbf{P}_{w}^{\mathrm{T}}\left(-\mathbf{D}_{B_{j}}(\phi(t))\right) \mathbf{P}_{w} \dot{\phi}_{j-3}(t)$, and defining

$$
\lambda_{D}=\sum_{j=4}^{n} \lambda_{D_{B j}, \max }
$$

we find that

$$
\begin{gathered}
\dot{V} \leq \boldsymbol{w}^{\mathrm{T}} \boldsymbol{g}_{1}+\frac{1}{2} \boldsymbol{w}^{\mathrm{T}}\left(\dot{\mathbf{M}}_{11}-2 \mathbf{P}_{w}^{\mathrm{T}} \mathbf{C P}_{w}\right) \boldsymbol{w}+ \\
\|\boldsymbol{w}\|\left\|\boldsymbol{k}_{1}\right\|+\|\boldsymbol{w}\|\left\|\boldsymbol{k}_{2}\right\|+\lambda_{D}\|\boldsymbol{w}\|^{2}
\end{gathered}
$$

The above expression (68) can be further simplified since

$$
\begin{aligned}
\frac{1}{2} \boldsymbol{w}^{\mathrm{T}}\left(\dot{\mathbf{M}}_{11}-2 \mathbf{P}_{w}^{\mathrm{T}} \mathbf{C} \mathbf{P}_{w}\right) \boldsymbol{w} & =\frac{1}{2} \boldsymbol{w}^{\mathrm{T}} \mathbf{P}_{w}^{\mathrm{T}}(\dot{\mathbf{M}}-2 \mathbf{C}) \mathbf{P}_{w} \boldsymbol{w} \\
& =0,
\end{aligned}
$$

where we have used that $\dot{\mathbf{M}}_{11}=\mathbf{P}_{w}^{\mathrm{T}} \dot{\mathbf{M}} \mathbf{P}_{w}$ and that $(\dot{\mathbf{M}}-2 \mathbf{C})$ is skew-symmetric. The latter property is proved in e.g. [15].

The remaining term $\boldsymbol{w}^{\mathrm{T}} \boldsymbol{g}_{1}$ in $\dot{V}$ arises from friction, see (31), and will now be investigated. We start by partitioning the complete friction term in (31) as

$$
\boldsymbol{g}=\boldsymbol{g}_{V}+\boldsymbol{g}_{C} \Longrightarrow \boldsymbol{g}_{1}=\boldsymbol{g}_{V_{1}}+\boldsymbol{g}_{C_{1}} \in \mathbb{R}^{3},
$$

where

$$
\boldsymbol{g}_{V_{1}}=\mathbf{P}_{w}^{\mathrm{T}} \boldsymbol{g}_{V}, \quad \boldsymbol{g}_{C_{1}}=\mathbf{P}_{w}^{\mathrm{T}} \boldsymbol{g}_{C}
$$

and the viscous friction $\boldsymbol{g}_{V}$ and Coulomb friction $\boldsymbol{g}_{C}$ are

$$
\begin{aligned}
& \boldsymbol{g}_{V}=\sum_{i=3}^{n}\left(\left(\mathbf{J}_{v_{F i}}\right)^{\mathrm{T}}{ }_{B_{3}} \boldsymbol{F}_{F_{i}}^{V}+\left(\mathbf{J}_{v_{R i}}\right)^{\mathrm{T}}{ }_{B_{3}} \boldsymbol{F}_{R_{i}}^{V}\right) \\
& \boldsymbol{g}_{C}=\sum_{i=3}^{n}\left(\left(\mathbf{J}_{v_{F i}}\right)^{\mathrm{T}}{ }_{B_{3}} \boldsymbol{F}_{F_{i}}^{C}+\left(\mathbf{J}_{v_{R i}}\right)^{\mathrm{T}}{ }_{B_{3}} \boldsymbol{F}_{R_{i}}^{C}\right) .
\end{aligned}
$$

By inserting, for the viscous friction force, the constitutive law (18), and employing the identities ${ }_{B_{3}} \boldsymbol{F}_{\xi_{i}}^{V}={ }_{B_{3}} \boldsymbol{F}_{\xi_{i}}^{P_{V}}$ and ${ }_{B_{3}} \boldsymbol{F}_{\xi_{i}}^{V}=\mathbf{R}_{B_{i} B_{i}}^{B_{3}} \boldsymbol{F}_{\xi_{i}}^{V}$ for $\xi_{i}=F_{i}, R_{i}$, we find that

$$
\boldsymbol{g}_{V}=-\sum_{i=3}^{n} \sum_{\xi_{i}=R_{i}, F_{i}} \frac{F_{N_{i}}}{2}\left(\left(\mathbf{J}_{v_{\xi i}}\right)^{\mathrm{T}} \mathbf{R}_{B_{i}}^{B_{3}} \mathbf{D}_{\mu_{V} B_{i}} \boldsymbol{v}_{\xi_{i}}\right) \text {. }
$$

Using $\phi$ and $\boldsymbol{\nu}$ in expression (22) for ${ }_{B_{i}} \boldsymbol{v}_{\xi_{i}}$ and inserting the result into $\boldsymbol{g}_{V}$ in (73), we get

$$
\boldsymbol{g}_{V}=-\sum_{i=3}^{n} \underbrace{\sum_{\xi_{i}=R_{i}, F_{i}}\left(\left(\mathbf{J}_{v_{\xi i}}\right)^{\mathrm{T}} \mathbf{R}_{B_{i}}^{B_{3}} \frac{F_{N_{i}}}{2} \mathbf{D}_{\mu_{V}}\left(\mathbf{R}_{B_{i}}^{B_{3}}\right)^{\mathrm{T}} \mathbf{J}_{v_{\xi i}}\right) \boldsymbol{\nu}}_{\mathbf{M}_{V_{i}}(\boldsymbol{\phi})} .
$$

We now find the pleasant fact that

$$
\mathbf{M}_{V}(\phi)=\sum_{i=3}^{n} \mathbf{M}_{V_{i}}(\phi)
$$

is positive definite. Intuitively, this is the case since $\mathbf{M}_{V}$ is the analogy to a mass matrix of interconnected point masses any any time-instant, and such a mass matrix is positive definite. We formulate $\boldsymbol{g}_{V_{1}}$ defined in (72) by using the expression for $\nu$ in (51) and inserting (77) in (76), then

$$
\boldsymbol{g}_{V_{1}}=-\mathbf{P}_{w}^{\mathrm{T}} \mathbf{M}_{V} \mathbf{P}_{w} \boldsymbol{w}-\mathbf{P}_{w}^{\mathrm{T}} \mathbf{M}_{V} \mathbf{P}_{\phi} \dot{\boldsymbol{\phi}}
$$

We now move on to the Coulomb friction term $\boldsymbol{g}_{C}$ since we have managed to extract $\boldsymbol{w}$ from $\boldsymbol{g}_{V_{1}}$. The Coulomb friction force $\boldsymbol{F}_{\xi_{i}}^{C}$ is found as in (15) and by inserting this into the expression for $\boldsymbol{g}_{C}$ in (74), we obtain

$\boldsymbol{g}_{C}=-\sum_{i=3}^{n} \frac{1}{\pi_{\xi_{i}}} \sum_{F_{i}, R_{i}}\left(\left(\mathbf{J}_{v_{\xi_{i}}}\right)^{\mathrm{T}} F_{N_{i}} \mathbf{R}_{B_{i}}^{B_{3}} \mathbf{D}_{\mu_{C}} \arctan \left(\frac{B_{i} \boldsymbol{v}_{\xi_{i}}}{\epsilon}\right)\right)$.

By employing the expressions above that constitute $\boldsymbol{g}_{1}$, we find from (68) that the function $\dot{V}$ can now be written

$$
\begin{array}{r}
\dot{V} \leq-\boldsymbol{w}^{\mathrm{T}} \mathbf{P}_{w}^{\mathrm{T}} \mathbf{M}_{V} \mathbf{P}_{w} \boldsymbol{w}+\boldsymbol{w}^{\mathrm{T}}\left(-\mathbf{P}_{w}^{\mathrm{T}} \mathbf{M}_{V} \mathbf{P}_{\phi} \dot{\boldsymbol{\phi}}\right)+ \\
\boldsymbol{w}^{\mathrm{T}} \boldsymbol{g}_{C_{1}}+\|\boldsymbol{w}\|\left(\left\|\boldsymbol{k}_{1}\right\|+\left\|\boldsymbol{k}_{2}\right\|\right)+\lambda_{D}\|\boldsymbol{w}\|^{2} .
\end{array}
$$

Define the vectors

$$
\begin{aligned}
\boldsymbol{k}_{3} & =-\mathbf{P}_{w}^{\mathrm{T}} \mathbf{M}_{V} \mathbf{P}_{\phi} \dot{\boldsymbol{\phi}} \\
k_{4} & =\max _{\boldsymbol{\phi}, B_{i}} \boldsymbol{v}_{\xi_{i}}\left\|\boldsymbol{g}_{C_{1}}\left(\boldsymbol{\phi}, B_{i} \boldsymbol{v}_{\xi_{i}}\right)\right\|,
\end{aligned}
$$

where $k_{4}>0$ is the maximum value for $\left\|\boldsymbol{g}_{C_{1}}\right\|$ for all possible combinations of $\phi$ and ${ }_{B_{i}} \boldsymbol{v}_{\xi_{i}}$. In addition, define the positive constant $0<\eta<1$ and the positive definite matrix

$$
\overline{\mathbf{M}}_{V}=\mathbf{P}_{w}^{\mathrm{T}} \mathbf{M}_{V} \mathbf{P}_{w} \in \mathbb{R}^{3 \times 3} .
$$

The matrix $\overline{\mathbf{M}}_{V}$ is positive definite since it is the upper left submatrix of the positive definite matrix $\mathbf{M}_{V}$. By using the identities (81)-(83) together with $\eta$ in (80), we obtain

$$
\begin{aligned}
\dot{V} \leq & -(1-\eta) \boldsymbol{w}^{\mathrm{T}} \overline{\mathbf{M}}_{V} \boldsymbol{w}-\eta \boldsymbol{w}^{\mathrm{T}} \overline{\mathbf{M}}_{V} \boldsymbol{w}+ \\
& \|\boldsymbol{w}\|\left(\left\|\boldsymbol{k}_{1}\right\|+\left\|\boldsymbol{k}_{2}\right\|+\left\|\boldsymbol{k}_{3}\right\|+k_{4}\right)+ \\
& \lambda_{D}\|\boldsymbol{w}\|^{2} .
\end{aligned}
$$

We notice from their respective expressions that $\left\|\boldsymbol{k}_{1}\right\|,\left\|\boldsymbol{k}_{2}\right\|$, $\left\|\boldsymbol{k}_{3}\right\|$, and $k_{4}$ are all bounded for bounded $\phi, \dot{\phi}$, and $\ddot{\phi}$. We are now close to satisfying the inequality (47) which we stated in the beginning of this section. Similarly to the inequality (46), we have that

$$
\lambda_{M_{V}, \min }\|\boldsymbol{w}\|^{2} \leq \boldsymbol{w}^{\mathrm{T}} \overline{\mathbf{M}}_{V} \boldsymbol{w}
$$

where $\lambda_{M_{V}, \min }$ is the infimum over all $t$ of the smallest eigenvalue of $\overline{\mathbf{M}}_{V}(\phi(t))$. Since $\overline{\mathbf{M}}_{V}(\phi(t))$ is continuous and positive definite for all $\phi(t)$, and since $\phi(t)$ belongs to 
a compact set for all $t$, then $\lambda_{M, \min }>0$. By introducing (85) into (84), we obtain

$$
\begin{gathered}
\dot{V} \leq- \\
\left\|(1-\eta) \lambda_{M_{V}, \min }\right\| \boldsymbol{w}\left\|^{2}-\eta \lambda_{M_{V}, \min }\right\| \boldsymbol{w} \|^{2}+ \\
\|\boldsymbol{w}\|\left(\left\|\boldsymbol{k}_{1}\right\|+\left\|\boldsymbol{k}_{2}\right\|+\left\|\boldsymbol{k}_{3}\right\|+k_{4}\right)+ \\
\lambda_{D}\|\boldsymbol{w}\|^{2} .
\end{gathered}
$$

Hence, the positive definite function $W(\boldsymbol{w})$ in (47) is

$$
W(\boldsymbol{w})=(1-\eta) \lambda_{M_{V}, \min }\|\boldsymbol{w}\|^{2},
$$

and we obtain

$$
\dot{V} \leq-W(\boldsymbol{w})
$$

for

$$
\eta \lambda_{M_{V}, \min }\|\boldsymbol{w}\|^{2} \geq k_{5}\|\boldsymbol{w}\|+\lambda_{D}\|\boldsymbol{w}\|^{2},
$$

where we have defined

$$
k_{5}=k_{5}(\phi, \dot{\phi}, \ddot{\phi})=\left\|\boldsymbol{k}_{1}\right\|+\left\|\boldsymbol{k}_{2}\right\|+\left\|\boldsymbol{k}_{3}\right\|+k_{4}
$$

to simplify the remaining calculation. The positive constant $\mu$ in (47) needs to be found such that (88) is satisfied. From (89) we find that

$$
\|\boldsymbol{w}\| \geq \frac{k_{5}(\phi, \dot{\phi}, \ddot{\phi})}{\eta \lambda_{M_{V}, \min }-\lambda_{D}} .
$$

Hence, we define

$$
\mu=\sup _{\phi, \ddot{\phi}, \ddot{\phi}} \frac{k_{5}}{\eta \lambda_{M_{V}, \text { min }}-\lambda_{D}}>0 .
$$

We know that $k_{5}>0$ since $k_{4}>0$. In addition, $k_{5}$ is bounded since $\left\|\boldsymbol{k}_{1}\right\|,\left\|\boldsymbol{k}_{2}\right\|,\left\|\boldsymbol{k}_{3}\right\|$, and $k_{4}$ are all bounded for $\phi, \dot{\phi}$, and $\ddot{\phi}$ bounded. We know that $\mu>0$ since $k_{5}>0$ and $\eta \lambda_{M_{V}, \min }>\lambda_{D}$ for $\dot{\phi}$ small enough since we see that $\lambda_{D}$ in (66)-(67) is proportional to $\dot{\phi}$ and we know from (85) that $\eta \lambda_{M_{V}, \text { min }}$ is independent of $\dot{\phi}$. Hence, we have that

$$
\dot{V} \leq-W(\boldsymbol{w}), \quad\|\boldsymbol{w}\| \geq \mu>0,
$$

which together with (46) proves that $\boldsymbol{w}$ is bounded. This concludes the proof of Theorem 1 .

\section{Simulation Results}

In this section, we present and compare the simulation results of a snake robot moving by lateral undulation where its joints are controlled by a standard PD-controller and the controller based on input-output linearization presented in this paper, respectively. We first implement the controllers on a model with the smooth approximation to the Coulomb friction. Then, we test the controllers on a model with setvalued Coulomb friction laws.

The simulation parameters are given in the following. Links $i=1,2$ have no mass or length since these are part of the virtual structure to give the global position and orientation of the head of the snake robot. For $i=3, \ldots, n$, we have $l_{i}=0.122 \mathrm{~m}, l_{f_{i}}=0.0457 \mathrm{~m}, m_{i}=7.5 / 11 \approx$ $0.682 \mathrm{~kg}$, and $\Theta_{i}=9.63 \times 10^{-4} \mathrm{~kg} \mathrm{~m}{ }^{2}$. The Coulomb friction parameters are $\mu_{C_{x}}=0.2, \mu_{C_{y}}=0.5$, and the viscous friction parameters are $\mu_{V_{x}}=0.1, \mu_{V_{y}}=0.2$. The controller gains for input-output linearization were $k_{D}=$ $2.4 \times 10^{3}$ and $k_{P}=400 \times 10^{3}$, where $k_{D}$ and $k_{P}$ are the elements on the diagonal in $\mathbf{K}_{D}$ and $\mathbf{K}_{P}$, respectively. To compare the simulation results, we have also implemented a PD-controller. For this controller, we set $d_{D}=1.2$ and
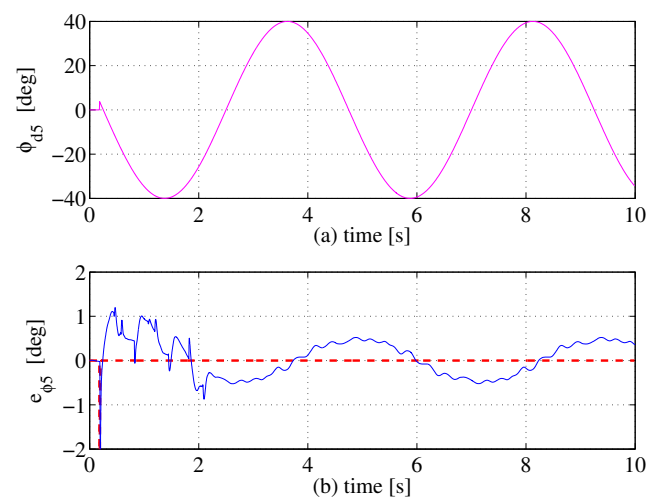

Fig. 2. Smooth friction law in model: (a) Desired joint angle for joint 5 , and (b) joint tracking error for the PD-controller (solid line) and the input-output linearization controller (thick dashed line).

$d_{P}=200$ as the elements on the diagonal matrices $\mathbf{D}_{D}$ and $\mathbf{D}_{P}$ respectively. The PD-controller is implemented as

$$
\boldsymbol{\tau}=-\mathbf{D}_{D}\left(\dot{\phi}-\dot{\phi}_{d}\right)-\mathbf{D}_{P}\left(\phi-\phi_{d}\right) .
$$

The simulations were performed in Matlab where the timestepping method (see [13], [18], [19]) was used for numerical integration of the model with set-valued Coulomb friction, while the solver ode15s was used for the smooth model.

The desired joint angles are found from (42) with the motion pattern parameters $A=40 \frac{\pi}{180}, \omega=80 \frac{\pi}{180} \frac{1}{\mathrm{~s}}$, and $\delta=-50 \frac{\pi}{180}$. We implement a 'soft-start' approach during start-up of the snake robot from its initial (straight) posture to avoid large steps in the reference signal. To this end, we set $\phi_{d_{j}}(t)=0$ until we have $\left|\phi_{d_{j}}(t)\right|<3 \frac{\pi}{180}, j=1, \ldots, n-3$, for the first time after start-up $(t=0)$. We choose to study one of the middle joints $\left(\phi_{5}\right)$ in the following to see how the controllers works for a joint that is strongly affected by the net snake motion due to the coupling with both ends of the snake robot.

\section{A. Smooth Coulomb Friction Model}

In this section, the smooth approximation (15) to the Coulomb friction has been employed in the mathematical model. Fig. 2 suggests that the input-output linearization controller yields an asymptotically stable error dynamics, while PD-controller results in a periodic tracking error.

\section{B. Set-Valued Coulomb Friction Model.}

In this section, the set-valued force law (16) was used as a constitutive law for the Coulomb friction. We see from Fig. 3 (b) that the tracking error for the controller based on input-output linearization is almost negligible despite that we no longer are able to cancel the snake robot dynamics correctly with the feedback term. The tracking error for the PD-controller is sinusoidal and almost similar in form but somewhat smaller in amplitude compared to the case with smooth Coulomb friction.

\section{Discussion of the Simulation Results.}

The snake robot is a nonlinear system and the simulation results confirms that a nonlinear controller is needed in order to control the snake robot joint angles accurately. We see from Fig. 2 and Fig. 3 that the proposed controller based on feedback linearization almost instantaneously controls the joint angle to its reference angle at start-up. In addition, 

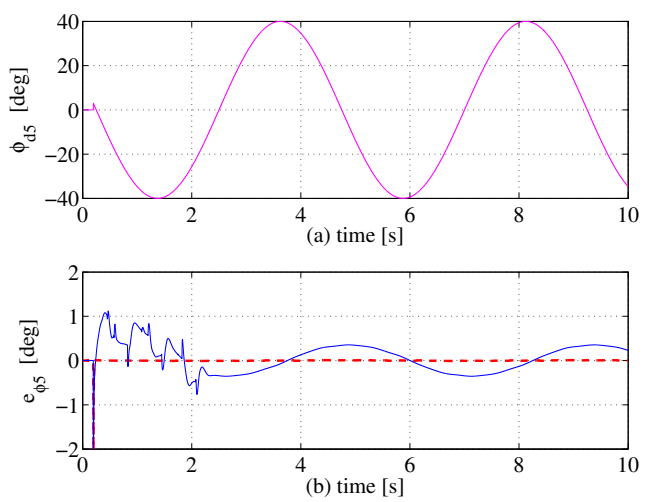

Fig. 3. Set-valued friction law in model: (a) Desired joint angle for joint 5 , and (b) joint tracking error for the PD-controller (solid line) and the input-output linearization controller (thick dashed line).

the tracking error is almost negligible for the non-smooth snake robot model. This suggests that the controller exhibits a relatively small sensitivity to errors in the friction model. This is a promising results since the friction may vary depending on which surface the snake robot is moving on and it may thus be difficult to estimate the friction.

It was difficult to tune the PD-controller gains and the PD-controller gave a much worse tracking error than for input-output linearization. This tuning problem was partly because the joints close to the ends can move much easier than the middle joints since the outer joints do not need to move both halves of the snake robot. Hence, the PDcontrollers should perhaps have been tuned independently for each joint. The chatter in the tracking error dynamics during the first two seconds for the PD-controller comes from that the remaining joints are still in the start-up process. Hence, the other joints affect the motion of joint 5 more when using the PD-controller.

\section{CONCLUSIONS AND FURTher WORK}

In this paper a control law for tracking control of the joints of a snake robot without wheels. Since the friction between the snake robot and the ground surface is essential for providing locomotion for wheel-less snake robots, a kinematic model is not sufficient, and a nonlinear model including both the kinematics and dynamics of the snake robots and its contact with the environment is presented. This model is denoted the process plant model. Furthermore, a control plant model is developed for the same snake robot. This model allows for an infinite number of rotations of the snake robot without affecting the controller. Based on this control plant model, a nonlinear controller is developed using input-output linearization. It is proved using Lyapunov stability theory that this controller asymptotically stabilizes the desired motion pattern for the snake robot joints. Furthermore, simulations with this controller applied to the process plant model suggest that the closed-loop system is able to track the desired joint angles. In addition, the same process plant model is implemented with a set-valued force law for describing the Coulomb friction instead of its smooth approximation. The simulations indicate that the proposed controller is also effective for the snake robot model with set-valued Coulomb friction.

In the future, we will try to include the velocity of the head of the snake robot in the stability proof and show that it can track a desired reference.

\section{REFERENCES}

[1] A. A. Transeth and K. Y. Pettersen, "Developments in snake robot modeling and locomotion," in Proc. IEEE Int. Conf. Control, Automation, Robotics and Vision, Dec 2006, pp. 1393-1400.

[2] S. Hirose, Biologically Inspired Robots: Snake-Like Locomotors and Manipulators. Oxford: Oxford University Press, 1993.

[3] P. Prautsch and T. Mita, "Control and analysis of the gait of snake robots," in Proc. IEEE Int. Conf. Control Applications, Kohala Coast, HI USA, 1999, pp. 502-507.

[4] J. Ostrowski and J. Burdick, "Gait kinematics for a serpentine robot," in Proc. IEEE Int. Conf. Robotics and Automation, vol. 2, April 1996, pp. 1294-1299.

[5] M. Saito, M. Fukaya, and T. Iwasaki, "Serpentine locomotion with robotic snakes," IEEE Contr. Syst. Mag., vol. 22, no. 1, pp. 64-81, February 2002

[6] S. Ma, "Analysis of creeping locomotion of a snake-like robot," $A d v$. Robotics, vol. 15, no. 2, pp. 205-224, 2001.

[7] G. Chirikjian and J. Burdick, "The kinematics of hyper-redundant robot locomotion," IEEE Transactions on Robotics and Automation, vol. 11 , no. 6, pp. 781-793, December 1995.

[8] J. Burdick, J. Radford, and G. Chirikjian, "A 'sidewinding' locomotion gait for hyper-redundant robots," in Proc. IEEE Int. Conf. Robotics and Automation, May 1993, pp. 101-106.

[9] S. Kelly and R. M. Murray, "Geometric phases and robotic locomotion,” J. Robotic Systems, vol. 12, no. 6, pp. 417-431, 1995.

[10] Ch. Glocker, Set-Valued Force Laws, Dynamics of Non-Smooth Systems, ser. Lecture Notes in Applied Mechanics. Berlin: SpringerVerlag, 2001, vol. 1.

[11] Ch. Glocker and C. Studer, "Formulation and preparation for numerical evaluation of linear complementarity systems in dynamics," Multibody System Dynamics, vol. 13, pp. 447-463, 2005.

[12] C. Le Saux, R. I. Leine, and Ch. Glocker, "Dynamics of a rolling disk in the presence of dry friction," Journal of Nonlinear Science, vol. 15, no. 1, pp. 27-61, 2005.

[13] A. A. Transeth, R. I. Leine, Ch. Glocker, and K. Y. Pettersen, "Non-smooth 3D modeling of a snake robot with frictional unilateral constraints," in Proc. IEEE Int. Conf. Robotics and Biomimetics, Dec 2006, pp. 1181-1188.

[14] H. Nijmeijer and A. v. d. Schaft, Nonlinear Dynamical Control Systems. New York: Springer-Verlag, 1990.

[15] M. W. Spong and M. Vidyasagar, Robot Dynamics and Control. Wiley \& Sons Inc., 1989

[16] P. Liljebäck, Ø. Stavdahl, and K. Y. Pettersen, "Modular pneumatic snake robot: 3D modelling, implementation and control," in Proc. 16th IFAC World Congress, July 2005.

[17] O. Egeland and J. T. Gravdahl, Modeling and Simulation for Automatic Control. Trondheim, Norway: Marine Cybernetics, 2002.

[18] A. A. Transeth, R. I. Leine, Ch. Glocker, and K. Y. Pettersen, "Nonsmooth 3D modeling of a snake robot with external obstacles," in Proc. IEEE Int. Conf. Robotics and Biomimetics, Dec 2006, pp. 1189-1196.

[19] R. I. Leine and H. Nijmeijer, Dynamics and Bifurcations of NonSmooth Mechanical Systems, ser. Lecture Notes in Applied and Computational Mechanics. Berlin: Springer Verlag, 2004, vol. 18.

[20] M. W. Spong, "Energy based control of a class of underactuated mechanical systems," in Proc. 13th IFAC World Congress, vol. F, July 1996, pp. 431-436.

[21] Y.-L. Gu and Y. Xu, "A normal form augmentation approach to adaptive control of space robot systems," in Proc. IEEE Int. Conf. Robotics and Automation, vol. 2, 1993, pp. 731-737.

[22] T. Kane and D. Lecison, "Locomotion of snakes: A mechanical 'explanation'," Int. J. Solids Struct., vol. 37, no. 41, pp. 5829-5837, October 2000.

[23] S. Ma, "Analysis of snake movement forms for realization of snakelike robots," in Proc. IEEE Int. Conf. Robotics and Automation, vol. 4, Detroit, MI USA, May 1999, pp. 3007-3013.

[24] H. K. Khalil, Nonlinear Systems, 3rd ed. Prentice Hall, 2000, international Edition. 